Western University

Scholarship@Western

Aboriginal Policy Research Consortium International (APRCi)

$1-21-2012$

\title{
Blunting the legacy of alcohol abuse in Western Australia
}

Tony Kirby

Follow this and additional works at: https://ir.lib.uwo.ca/aprci

Part of the Community Health Commons, Public Health Education and Promotion Commons, and the Substance Abuse and Addiction Commons

Citation of this paper:

Kirby, Tony, "Blunting the legacy of alcohol abuse in Western Australia" (2012). Aboriginal Policy Research Consortium International (APRCi). 415.

https://ir.lib.uwo.ca/aprci/415 


\title{
Blunting the legacy of alcohol abuse in Western Australia
}

\author{
After bravely ushering in alcohol sale restrictions proposed by its residents, a remote Western \\ Australian community is tackling the long-term effects of alcohol misuse. Tony Kirby reports.
}

Fitzroy Crossing, an isolated town with approximately 2500 residents in Australia's remote northwest, was typical of such communities, with a large Indigenous population (around $60 \%$ ), high rates of harmful alcohol use, and depressingly high rates of alcohol-related violence and crime.

A tipping point came in 2007 when there were 55 deaths in the community, 13 being suicides. Alcohol was a factor in most deaths. After much consultation, community elders took what at the time was unthinkable action: proposing that all stores selling take-away alcohol be restricted only to low-strength beverages, with a maximum of $2.5 \%$ alcohol by volume. Take-away sales of stronger beers or ciders, wine, and spirits were prohibited, meaning these could only be bought in the bar of the town's two licensed premises that were prohibitively expensive to many residents. The nearest town selling take-away high strength alcohol is $250 \mathrm{~km}$ away. Despite stern opposition and personal threats to those leading the reform, there was sufficient community support to implement a trial of the restrictions.

12 months after their introduction, independent assessment showed a 28\% reduction in alcohol-related incidents attended by police, a $42 \%$ reduction in alcohol-related hospital presentations, and a $14 \%$ increase in school attendance. Fitzroy Crossing suddenly became a more pleasant place to live and work. Local police could engage proactively with the community, rather than responding to incidents over which they had no control. So successful were the restrictions that the West Australian Liquor Licensing authorities extended them indefinitely.

Alcohol can cause a range of permanent physical and neurocognitive abnormalities known as fetal alcohol spectrum disorders (FASD), including specific diagnoses of fetal alcohol syndrome (FAS), partial FAS, and neurodevelopmental disorder-alcohol exposed (ND-AE). "Alcohol consumption during pregnancy can cause birth defects, growth restriction, and a range of lifelong developmental,

\section{"Preliminary data from the Lililwan Project suggest that about $50 \%$ of Indigenous women in the Fitzroy Valley drink during pregnancy..."}

neurological, and behavioural problems", says paediatrician Elizabeth Elliott, one of Australia's leading FASD experts and a chief investigator on the Lililwan Project-Australia's first population-based prevalence study on FASD. "FAS is characterised by obvious physical manifestations: a smooth philtrum (the divot or groove between the nose and upper lip); a thin upper lip; and narrow eye openings. But there are many more children exposed to alcohol who have neurodevelopmental disorder without physical features, making diagnosis difficult in this group", she adds.

In surveys of non-Indigenous Australian women, $60 \%$ report drinking in pregnancy, and $4 \%$ report drinking at binge levels of five or more standard drinks per occasion during pregnancy. By contrast, $20 \%$ of Indigenous women report drinking in pregnancy. Although surveys suggest that Indigenous Australian women are twice as likely than non-Indigenous women to drink at high-risk levels, there are limited data in the Indigenous population on drinking at risky levels in pregnancy. Preliminary data from the Lililwan Project suggest that about $50 \%$ of
Indigenous women in the Fitzroy Valley drink during pregnancy and that as many as nine in ten of those women who do drink in pregnancy do so at high-risk levels.

Although the alcohol restrictions will help future generations, Fitzroy Crossing faces a long-term effect of endemic alcohol abuse: FASD among their children. In 2008, a FASD leadership team including local organisations and service providers formed to develop the Marulu strategy to address FASD. The strategy includes diagnosis and prevention, community education, support for those affected, as well as the Lililwan Project.

"FASD is a tragic legacy of society's careless relationship with alcohol", says James Fitzpatrick, paediatric senior registrar, PhD student, and a Lililwan Project chief investigator. "The period of fetal development is an opportunity to provide optimal conditions for brain development. The factors leading to consumption of alcohol during this period undermine this opportunity and the result is too often the permanent neurological and behavioural sequelae of FASD", he adds.

The Lililwan Project is a partnership between Nindilingarri Cultural Health

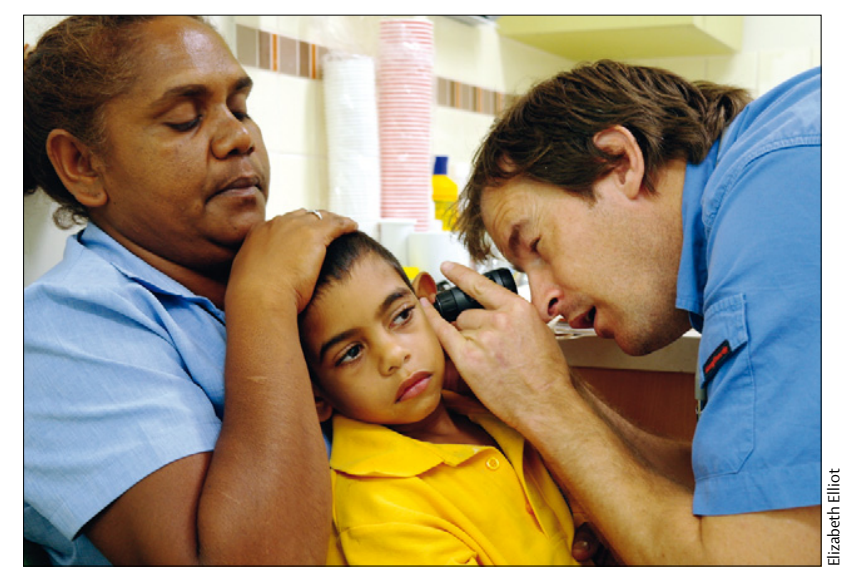

James Fitzpatrick undertakes a child health assessment in a remote community 
Services, Marninwarntikura Women's Resource Centre (MWRC) (both at Fitzroy Crossing), the George Institute for Global Health, Sydney, and the Discipline of Paediatrics and Child Health at the University of Sydney. The first stage involved interviewing the parent or carer of all children born in 2002 and 2003 and residing in the Fitzroy Valley to describe the demographics, antenatal exposures (including alcohol), birth, and health history of the cohort. Stage two, now nearing completion, involves multidisciplinary clinical examination of these children and is funded by the Australian Government.

"Before commencing this study, Community Navigators (local Aboriginal Health Workers on the project) visited over 45 outlying remote communities to explain the study to parents and gain consent", says Jane Latimer, Senior Research Fellow at the George Institute, Associate Professor at the University of Sydney Medical School, and also one of the Lililwan chief investigators. "Over $95 \%$ of eligible children and their parents consented to participate, reflecting the support for the project from the community. Each child in the study receives a health and developmental assessment identifying their strengths and needs and all parents have been provided with verbal feedback and a written management plan to address problems identified", adds Latimer.

"FASD is a condition that these children will be living with from birth to grave", says June Oscar, a chief investigator on the project and head of MWRC. "Any support required is lifelong. The key message is that the child with FASD requires others to become aware, and change their attitudes and thinking so that those affected can benefit and be the best that they can be. Individuals with FASD remind us that we need solutions that are diverse and that one size does not necessarily fit all." To further highlight the issues around FASD, the Lililwan Project team will produce Tristan's Story, a documentary about a child with FASD living in the Fitzroy Valley. Australia's
Governor General will launch the film in Australia, before the Australian Human Rights Commission screens it at the UN Permanent Forum for Indigenous Peoples in New York in May, 2012.

Targeted FASD interventions include teaching caregivers techniques to calm affected children, and teachers modifying the classroom to minimise excessive words and pictures on walls that could distract a child with attention problems. "For at-risk young children with prenatal alcohol exposure, preventive interventions are needed", says Heather Olson, a psychologist and FASD expert at the University of Washington, Seattle, WA, USA who has worked on the Lililwan Project. Olson adds that it is essential caregivers are trained correctly. "Early intervention should then aim to concurrently reduce the impact of disabling child characteristics and cumulative environmental risk while at the same time enhancing protective factors." Oscar adds that "Patience, understanding, repetition, simplicity, love, and care are some of the critical elements of responding to and managing someone with FASD."

Australia's Aboriginal and Torres Strait Islander Social Justice Commissioner, Mick Gooda, described the project as "a genuine partnership-where research is done with the community and not just about the community; setting an example to the rest of Australia as a process guided by meaningful, respectful engagement and collaboration".

"The biggest difference with our action to pursue alcohol restrictions is that it was a community driven intervention to the situation at the time", says Oscar. "We knew what we needed governments to do in support of our decision. Strategies such as these can only work if the community leadership is able to support it at every level: individual, family, community, organisational. Local stakeholders have to be leading it from the local level with the support of governments."
Local volunteer Meredith Kefford helped lead the community consultation that preceded the prevalence study. "We did not shy away from discussing the potential risks of such a study-further stigmatisation, lack of meaningful ongoing support after diagnosis, blaming and shaming", says Kefford. "But people were overwhelmingly concerned about helping the children-they knew many of them were struggling Some mothers were expecting the FASD diagnosis and in some ways were relieved to have an explanation and some support for their child The project included counselling for parents and carers of children diagnosed with a FASD." Kefford hopes the alcohol restrictions remain in place as they help protect some of the most vulnerable people in the community.

Oscar is hopeful other communities can learn from Fitzroy Crossing. "Our experience shows initiatives like this are possible, and that there is hope for a future and a life well lived by all. But local stakeholders have to lead projects like this from the local level with the support of governments." Kefford agrees, adding: "I have no doubt that the decision to go ahead with the study, and the subsequent high participation rates were because this was a community initiative run by community organisations-it could never have happened if driven externally."

In 2011, the Australian Government announced an inquiry into FASD incidence and prevention, including targeting prevention solutions for high-risk groups such as Indigenous communities and teenage-binge drinkers. "The recommendations from this inquiry will be used by government to formulate policy around this issue", says Latimer. She hopes that the huge positives from the Lililwan Project can be successfully transferred to other communities in Australia that have, like Fitzroy Crossing, faced an intolerable burden of alcohol-related ill health for too long.

Tony Kirby 